\title{
Barriers to Primary Care Physicians Prescribing Buprenorphine
}

\author{
Eliza Hutchinson, BA \\ Mary Catlin, BSN, MPH \\ C. Holly A. Andrilla, MS \\ Laura-Mae Baldwin, MD, MPH \\ Roger A. Rosenblatt, MD, MPH, \\ MFR
}

University of Washington, Department of Family Medicine, Research Section, Seattle, Washington

\begin{abstract}
PURPOSE Despite the efficacy of buprenorphine-naloxone for the treatment of opioid use disorders, few physicians in Washington State use this clinical tool. To address the acute need for this service, a Rural Opioid Addiction Management Project trained 120 Washington physicians in 2010-2011 to use buprenorphine. We conducted this study to determine what proportion of those trained physicians began prescribing this treatment and identify barriers to incorporating this approach into outpatient practice.
\end{abstract}

METHODS We interviewed 92 of 120 physicians (77\%), obtaining demographic information, current prescribing status, clinic characteristics, and barriers to prescribing buprenorphine. Residents and 7 physicians who were prescribing buprenorphine at the time of the course were excluded from the study. We analyzed the responses of the 78 remaining respondents.

RESULTS Almost all respondents reported positive attitudes toward buprenorphine, but only $22(28 \%)$ reported prescribing buprenorphine. Most $(95 \%$, $n=21$ ) new prescribers were family physicians. Physicians who prescribed buprenorphine were more likely to have partners who had received a waiver to prescribe buprenorphine. A lack of institutional support was associated with not prescribing the medication $(P=.04)$. A lack of mental health and psychosocial support was the most frequently cited barrier by both those who prescribe and who do not prescribe buprenorphine.

CONCLUSION Interventions before and after training are needed to increase the number of physicians who offer buprenorphine for treatment of addiction. Targeting physicians in clinics that agree in advance to institute services, coupled with technical assistance after they have completed their training, their clinical teams, and their administrations is likely to help more physicians become active providers of this highly effective outpatient treatment.

Ann Fam Med 2014;128-133. doi:10.1370/afm.1595.

\section{INTRODUCTION}

I n 2000, the passage of the Drug Addiction Treatment Act enabled qualifying physicians to receive a waiver from the special registration requirement of the Controlled Substances Act to prescribe buprenorphine-naloxone for the treatment of opioid use disorders. Buprenorphinenaloxone, hereafter referred to as buprenorphine, is the only treatment available to office-based physicians that has high rates of prolonged success for the treatment of opioid addiction. ${ }^{1,2}$ Although physicians have reported a wide range of barriers that have dissuaded them from prescribing buprenorphine, ${ }^{3-16}$ few studies specifically addressed the barriers family practice physicians encounter. McCarty et a ${ }^{10}$ found that 6 of 17 Oregonian family physicians trained to use buprenorphine prescribe it, whereas those who do not prescribe buprenorphine cited concerns of being overwhelmed by needy patients, the cost of medication, reimbursement, being the only physician prescribing buprenorphine in the practice, and lack of administrative support. Even though Walley et $\mathrm{al}_{1}{ }^{15}$ in his survey of physicians who had received a waiver to prescribe buprenorphine 
in Massachusetts, did not analyze barriers by specialty, his sample included 66 (44\%) family practitioners. Physicians who were prescribing buprenorphine reported a lack of institutional, nursing, and office support, whereas the physicians not prescribing buprenorphine cited these obstacles, plus a lack of staff knowledge and lack of patient need. Data from national surveys ${ }^{4}$ report an increasing proportion of family physicians among the Drug Enforcement Agency list of all physicians who had received waivers-26\% in 2008; survey limitations were that fewer physicians not prescribing buprenorphine responded to the survey, and some response rates were less than $29 \%$. Thus there is lack of certainty about which barriers to address to expand access to opioid treatment in primary care.

In 2009 the Rural Opioid Addiction Management Project was established in Washington State to address the epidemic of addiction to prescription opioids. Of the 39 counties in Washington State, 14 had no physicians who had received a waiver to prescribe buprenorphine, and one objective of the project was to add buprenorphine prescribers to the 35 rural and 360 urban physicians who had received waivers. The project also focused on training physicians in locations adjacent to rural areas because there was a shortage of rural physicians. Physicians were recruited by contacting clinicians and administrators, and facilities were visited in areas with high opioid death rates. Information about prescription drug misuse, buprenorphine, and the upcoming authorized training courses on the treatment and management of opioid-dependent patients was publicized widely. After the recruited physicians completed 1 of the first 5 courses offered, we undertook this study to determine the barriers the trained physicians encountered as they considered prescribing buprenorphine and how many had begun to treat opioid addiction.

\section{METHODS}

\section{Sample}

Our study includes physicians who trained between October 30, 2010, and November 5, 2011. The study began 7 months after the November course to allow sufficient time for the physicians to receive a waiver and begin prescribing buprenorphine.

\section{Survey Process and Instrument}

The senior investigator trained 2 medical student interviewers and tested the semistructured, 10 -minute questionnaire during the pilot interviews of 6 physicians who had received waivers. We purchased a Drug Enforcement Agency (DEA) list to verify waiver status.

For the study, the interviewers e-mailed or telephoned physicians, and those who agreed were admin- istered the questionnaire. The interviewers recorded responses to open-ended questions by taking notes. The University of Washington Institutional Review Board approved this study.

\section{Variables}

Interviewers asked about demographic information, practice characteristics, and the perceived barriers to providing office-based treatment. Respondents reported their specialty affiliations in an open-ended question and indicated whether they worked in single or multispecialty practices.

To learn about barriers that prevented physicians from prescribing buprenorphine, interviewers asked, "I'll mention a list of items, and please say 'yes' if they have posed a barrier to you in proving buprenorphine treatment, or 'no' if they have not posed a barrier." After the respondents replied to the list of possible barriers, they were then asked, "Did you encounter any other barriers that I have not mentioned?" and were invited to elaborate.

Respondents were asked to note their clinical opinion of the effectiveness of buprenorphine as a treatment for addiction on a scale of 1 to 5 , with 1 being very unfavorable and 5 , very favorable.

\section{Data Analysis}

The frequency of variables was described and crosstabulated by prescriber status. $t$ Tests, $\chi^{2}$ tests, and Fisher's exact tests were used to determine the significance of between-group differences.

\section{Specialty, Rural-Urban Location}

We aggregated the specialties into family medicine and other specialties. Participants were listed as rural or urban based on the Health Resources and Services Administration classification of their county of residence (http://datawarehouse.hrsa.gov/RuralAdvisor/ RuralHealthAdvisor.aspx).

\section{RESULTS}

Of 120 physicians who completed the training, 92 responded to the questionnaire $(77 \%)_{i} 28$ physicians did not respond -5 declined, and 23 did not return our telephone calls after 2 to 5 attempts. There were no significant differences by sex, urban practice location, or the proportion who obtained a waiver between the respondents and nonrespondents. We further excluded 7 of the physicians who were in residency programs and 7 others who were prescribing buprenorphine before the training course, leaving 78 respondents for analysis.

Of these 78 physicians, 50 obtained the DEA waiver (64\%) to prescribe buprenorphine. Respondents 
were grouped into physicians who had prescribed buprenorphine to 1 or more patients since completing the course $(\mathrm{n}=22)$ and physicians who had not $(\mathrm{n}=56)$.

\section{Respondent Characteristics}

Characteristics of the 78 physicians are shown in Table 1 . They were predominantly doctors of medicine $(91 \%, \mathrm{n}=71)$, male $(60 \%, \mathrm{n}=47)$, and had a mean age of 52.1 years (range of 30 to 82 years). Most were employed in single-specialty clinics $(65 \%, \mathrm{n}=51)$ in urban counties $(69 \%, n=54)$, in a variety of practice types. Practices in which physicians began prescribing buprenorphine had mean sizes of 9.0 physicians compared with 13.9 physicians for those not prescribing buprenorphine, but the difference was not statistically significant.

The characteristics associated with trained physicians who prescribed buprenorphine are shown in Table 2. Twenty-one of the 22 physicians who began to prescribe buprenorphine were family physicians;
1 was a psychiatrist. Overall, family physicians were more than 5 times more likely to prescribe buprenorphine than trained physicians from other specialties: $33 \%$ (21 of 63 ) vs $7 \%$ ( 1 of 15 ; Fisher's exact test, 2 -sided, $P=.05$ ). None of the 4 internists had begun to prescribe buprenorphine by the time of the survey.

Having another physician with a waiver in the practice was significantly associated with becoming a prescriber of buprenorphine. Fifteen of the 22 (68\%) who prescribed buprenorphine after training reported having at least 1 partner with a waiver, although only 12 partners actually prescribed buprenorphine.

\section{Number of Patients Treated and Acceptance of Patents for Care of Prescribing Physicians}

Table 3 shows the varied experiences of 22 physicians prescribing buprenorphine. Prescribers reported having treated between from 1 to 50 patients since receiving their waiver. One-half $(50 \%, \mathrm{n}=11)$ of these newly trained physicians reported having treated 3 or fewer patients. Newly trained physicians were cautious in the selection of patients they would treat for opioid-related disorders. Ninety-five percent ( $\mathrm{n}=21)$ would prescribe treatment for their current patients. Three of the 22 physicians were in solo practice; of the remaining 19 physicians, $89 \%$ $(\mathrm{n}=17)$ would treat patients from their practice partners. Most of the 22 physicians $(73 \%, \mathrm{n}=16)$ would accept referred patients or patients from other practices. Most would also accept patients from their community who were outside the practice $(64 \%, \mathrm{n}=14)$. Only $36 \%$ $(\mathrm{n}=8)$ would accept patients from other communities, even though 10 of 39 counties had neither a buprenorphineprescribing physician nor a methadone clinic. Fewer than one-half of the physicians $(41 \%, n=9)$ listed their name on the Substance Abuse and Mental Health Administration's (SAMHSA) Physician and Treatment Locator website (http://buprenorphine.samhsa. gov/bwns_locator/). 
Of the 69 trained physicians who offered their clinical opinion of the effectiveness of buprenorphine, the average was a favorable rating of 4.3 (1= very unfavorable, $5=$ very favorable), and $92 \%$ of all respondents ( $\mathrm{n}$ $=72,5$ offered no comment) would recommend it for the treatment of opioid addiction. There was no difference between those who prescribed and those who did not prescribe buprenorphine.

\section{Barriers to Prescribing Buprenorphine for Opioid Addiction Treatment}

Figure 1 compares the perceived barriers to prescribing buprenorphine reported by those who do and do not prescribe it. Physicians not prescribing were significantly more likely to cite a lack of institutional support. In contrast to prior studies, respondents most frequently reported the lack of mental health and psychosocial support as an obstacle $(64 \%, \mathrm{n}=50)$. Respondents also cited time constraints $(54 \%, \mathrm{n}=42)$, lack of specialty backup $(45 \%, n=35)$, lack of confidence in their ability to manage opioid addiction $(41 \%, \mathrm{n}=32)$, resistance from practice partners $(42 \%, \mathrm{n}=33)$, and lack of institutional support $(36 \%, \mathrm{n}=28)$. Contrary to our expectation, relatively few mentioned concerns about financial issues $(28 \%, \mathrm{n}=22)$.

When asked what could facilitate prescribing, 9 respondents mentioned the local telemedicine access to specialists but requested the service at times that did not conflict with patient care. Ten respondents suggested a follow-up course or site visits after training to help them with implementation.

\section{DISCUSSION}

Translating Training into Practice Remains Slow There were 78 physicians who were trained in these courses, and 50 obtained the DEA waiver to prescribe buprenorphine, but only 22 began to treat opioid addiction in the 7 to 18 months after training. Others have found that one-half to two-thirds of specialist physicians who have obtained a waiver to prescribe buprenorphine prescribe it. ${ }^{9,15}$ Some persons may have taken the course only to satisfy a new state pain education requirement, or Washington State's lower rate of initiating services may reflect the differences between voluntary early adopters ${ }^{17}$ compared with more recent recruited trainees. Some physicians may decide to prescribe in the future, as physicians in a national survey who obtained waivers were more likely to prescribe buprenorphine as time passed. ${ }^{9}$

\section{Barriers to Prescribing Cited by Physicians}

The need for behavioral health services is commonly cited as a barrier to prescribing buprenorphine, ${ }^{6,8,11,18}$ and in our study this need was cited most frequently by both physicians who do and do not prescribe buprenorphine. In Washington State, Medicaid pays for buprenorphine treatment only if clients receive chemical dependency counseling despite the lack of data that additional counseling improves outcomes. ${ }^{19}$ Physicians' requests for counseling may reflect the need for help treating severe mental illness, as well as the reality that counseling is needed to ensure reimbursement. 
Table 3. Experience and Satisfaction With Buprenorphine Reported by Prescribing Physicians

\begin{tabular}{lc}
\hline & $\begin{array}{c}\text { Prescribes } \\
\text { Buprenorphine } \\
(\mathbf{n}=\mathbf{2 2}) \\
\text { No. (\%) }\end{array}$ \\
\hline Patients ever treated with buprenorphine & 10.1 \\
Mean & $11(50)$ \\
$1-3$ & $6(27)$ \\
$4-10$ & $4(18)$ \\
$>10$ & $1(5)$ \\
Unknown & $9(41)$ \\
Has name on public list of prescribing physicians & \\
Clinical opinion of buprenorphine's effectiveness for treating & \\
opioid addiction & 4.3 \\
Mean score & $3(14)$ \\
Dissatisfied (1-3) & $18(82)$ \\
Satisfied (4-5) & $1(5)$ \\
Missing & \\
Types of patients physician is willing to accept for treatment & $21(95)$ \\
Patients already on personal panel & $17(89)^{\mathrm{a}}$ \\
Patients of other physicians in clinic & $16(73)$ \\
Referrals from other physicians or agencies & $14(64)$ \\
Patients from the community not in the practice & $8(36)$ \\
Patients from other communities not already in the practice & $8(36)$ \\
Has participated in telemedicine sessions offering addiction, & \\
pain, and psychiatry specialists & \\
\hline a Of 19 physicians, 3 prescribers were solo practitioners. & \\
\hline
\end{tabular}

Access to specialists was not cited as frequently $(45 \%, 35$ of 78$)$, perhaps because a weekly telemedicine service links physicians to specialists in psychiatry, addiction medicine, and pain. Institutional barriers were significantly associated with not prescribing buprenorphine, ${ }^{14,15}$ but in our group they were not common issues for prescribers.

Netherland et $\mathrm{al}^{11}$ and Arfken et $\mathrm{a}^{4}$ found that reimbursement was one of the most frequently noted barriers. In contrast, reimbursement was not a frequently cited concern of physicians who did and did not prescribe buprenorphine in our study. Concerns about reimbursement and drug costs may increase, however, unless buprenorphine becomes a pharmaceutical benefit covered under health care reform. Then, as now, covered benefits for effective treatment will vary by state.

This study has several limitations. Our results apply only to Washington State physicians newly trained in 1 of the first 5 courses offered by the Rural Opioid Addiction Management Project to train physicians to prescribe buprenorphine.

\section{Figure 1. Barriers to prescribing buprenorphine by prescribing and nonprescribing physicians.}

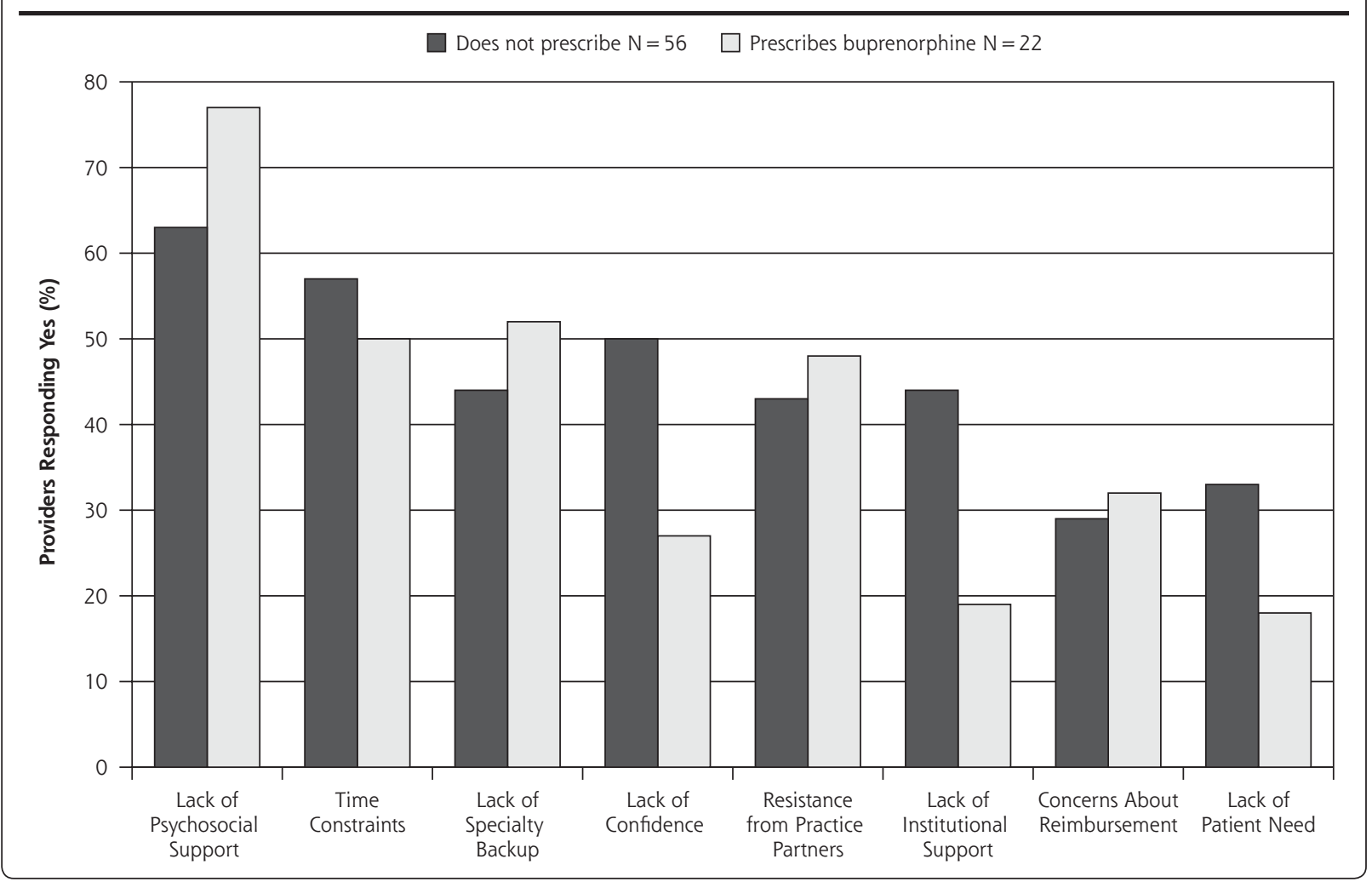


We also recruited rural physicians more heavily than urban physicians.

Our findings suggests that more than a training course is needed to convert trainees and primary care clinics into service providers in areas that have no access to buprenorphine. Before taking a course in prescribing buprenorphine, it may be wise to obtain an administrative commitment to provide treatment for addiction and to train providers in teams, thus facilitating both coverage and consultation. After training, technical assistance could help remove the remaining barriers to implementing care. Convenient access to specialists may increase the willingness to prescribe. Finally, the discussions currently underway to define which substance abuse treatments will be funded under health care reform will also remove or institute new barriers.

To read or post commentaries in response to this article, see it online at http://www.annfammed.org/content/12/2/128.

Key words: buprenorphine; opiate substitution treatment; rural health; primary health care; opiate addiction

Submitted April 18, 2012; submitted, revised, July 29, 2013; accepted August 14, 2013.

Funding support: The American Osteopathic Academy of Addiction Medicine and American Academy of Addiction Psychiatry sponsored the training courses, which were made possible in part, by contract \#IH79T1022022 from Substance Abuse and Mental Health Administration. We gratefully acknowledge funding from the Washington State Life Science Discovery Fund (WSU-LSDF subcontract number 109212G002562, PI John Roll).

Disclaimer: The contents of this article are the responsibility of the authors and do not reflect those of the funding agencies.

Acknowledgments: We would like to thank Martha Reeves for help formatting the manuscript, medical student Kelly Dundon for assistance with the interviews.

\section{References}

1. Fiellin DA, O'Connor PG. Clinical practice. Office-based treatment of opioid-dependent patients. N Engl J Med. 2002;347(11):817-823.

2. National Consensus Development Panel on Effective Medical Treatment of Opiate Addiction. Effective medical treatment of opiate addiction. JAMA. 1998;280(22):1936-1943.

3. Albright J, Ciaverelli R, Essex A, Tkacz J, Ruetsch C. Psychiatrist characteristics that influence use of buprenorphine medicationassisted treatment. J Addict Med. 2010;4(4):197-203.
4. Arfken $C L$, Johanson CE, di Menza S, Schuster CR. Expanding treatment capacity for opioid dependence with office-based treatment with buprenorphine: National surveys of physicians. J Subst Abuse Treat. 2010;39(2):96-104.

5. Barry DT, Irwin KS, Jones ES, et al. Integrating buprenorphine treatment into office-based practice: a qualitative study. J Gen Intern Med. 2009;24(2):218-225.

6. Cunningham CO, Kunins HV, Roose RJ, Elam RT, Sohler NL. Barriers to obtaining waivers to prescribe buprenorphine for opioid addiction treatment among HIV physicians. J Gen Intern Med. 2007;22(9): 1325-1329.

7. Ducharme LJ, Abraham AJ. State policy influence on the early diffusion of buprenorphine in community treatment programs. Subst Abuse Treat Prev Policy. 2008;3:17.

8. Gordon AJ, Kavanagh G, Krumm M, et al. Facilitators and barriers in implementing buprenorphine in the Veterans Health Administration. Psychol Addict Behav. 2011;25(2):215-224.

9. Kissin W, McLeod C, Sonnefeld J, Stanton A. Experiences of a national sample of qualified addiction specialists who have and have not prescribed buprenorphine for opioid dependence. J Addict Dis. 2006;25(4):91-103.

10. McCarty D, Rieckmann T, Green C, Gallon S, Knudsen J. Training rural practitioners to use buprenorphine; using The Change Book to facilitate technology transfer. J Subst Abuse Treat. 2004;26(3): 203-208.

11. Netherland J, Botsko M, Egan JE, et al; BHIVES Collaborative. Factors affecting willingness to provide buprenorphine treatment. J Subst Abuse Treat. 2009;36(3):244-251.

12. Roman PM, Abraham AJ, Knudsen HK. Using medication-assisted treatment for substance use disorders: evidence of barriers and facilitators of implementation. Addict Behav. 2011;36(6):584-589.

13. Schackman BR, Merrill JO, McCarty D, Levi J, Lubinski C. Overcoming policy and financing barriers to integrated buprenorphine and HIV primary care. Clin Infect Dis. 2006;43(Suppl 4):S247-S253.

14. Thomas CP, Reif S, Haq S, Wallack SS, Hoyt A, Ritter GA. Use of buprenorphine for addiction treatment: perspectives of addiction specialists and general psychiatrists. Psychiatr Serv. 2008;59(8): 909-916.

15. Walley AY, Alperen JK, Cheng DM, et al. Office-based management of opioid dependence with buprenorphine: clinical practices and barriers. J Gen Intern Med. 2008;23(9):1393-1398.

16. West JC, Kosten TR, Wilk J, et al. Challenges in increasing access to buprenorphine treatment for opiate addiction. Am J Addict. 2004; 13(Suppl 1):S8-S16.

17. Quest TL, Merrill JO, Roll J, Saxon AJ, Rosenblatt RA. Buprenorphine therapy for opioid addiction in rural Washington: the experience of the early adopters. J Opioid Manag. 2012;8(1):29-38.

18. Turner BJ, Laine C, Lin YT, Lynch K. Barriers and facilitators to primary care or human immunodeficiency virus clinics providing methadone or buprenorphine for the management of opioid dependence. Arch Intern Med. 2005;165(15):1769-1776.

19. Friedmann PD, Schwartz RP. Just call it "treatment". Addict Sci Clin Pract. 2012;7(1):10. 